\title{
Worrying data on excess mortality and comorbidity in MS
}

Levels of mortality and several comorbidities are increased in patients with multiple sclerosis (MS), according to a large US-based cohort study. The findings should alert the medical community to the necessity of close surveillance in patients with MS, as targeted interventions could reduce the burden of comorbidities.

\section{Early recognition and management of comorbidities ... is paramount... 77}

Drawing from an administrative claims database and the National Death Index, Gorana Capkun and colleagues assessed all-cause mortality and over 200 comorbidities in 15,684 patients with MS and 78,420 controls without MS (matched for age, sex and start of follow-up period). In line with previous reports, all-cause mortality was 2.9-fold higher in the MS than in the non-MS cohort.

Death from infectious disease (mortality rate ratio [MRR] 6.2, 95\% CI 4.2-9.4) was one of the driving causes of excess mortality in the MS cohort. Deaths from diseases of the nervous system other than MS, or from respiratory or circulatory system diseases, were also more common in the MS cohort than in the non-MS cohort. Moreover, risks of certain comorbid conditions, including ischaemic stroke, certain cancers, and ulcerative colitis, were increased in patients with MS.

Completed and attempted suicide and psychiatric disorders were also more common in patients with MS. The increase in deaths from suicides was particularly striking in young patients (18-29 years; MRR 10.1, 95\% CI 1.1-97.4), although this group was small.

"Early recognition and management of comorbidities in individuals with MS is paramount, and can potentially reduce premature mortality and improve quality of life," comments Capkun on the results. "Early treatment with efficacious medication could diminish damage arising from comorbidities and reduce the risk of premature death."
Further research is needed to establish the extent to which the findings by Capkun et al. could be attributed to MS-related pathogenetic processes and the use of immunomodulatory diseasemodifying therapies (DMTs). The researchers also suggest that insights from the study could be used as a baseline reference with which safety profiles of new DMTs could be compared.

Capkun notes that lower socioeconomic status or lifestyle-related factors in patients with MS could also influence their susceptibility to comorbidities. Such factors are potentially modifiable, and their identification could help prevent premature deaths.

Hemi Malkki

Original article Capkun, G. et al. Mortality and comorbidities in patients with multiple sclerosis compared with a population without multiple sclerosis: an observational study using the US department of defense administrative claims database. Mult. Scler. Relat. Disord. doi:10.1016/i.msard.2015.08.005 